\title{
El fenómeno de la violencia entre iguales en España
}

Roles, género, edad, actitudes y estrategias de intervención

Concepción Castro Clemente Doctoranda (Universidad Pontificia

Comillas Madrid, España)

Máster Oficial (Universidad Pontificia

Comillas Madrid, España)

Profesora-Tutora Grado Trabajo Social

(UNED), Madrid, España

Diplomada Trabajo Social (UNED), España Graduada Trabajo Social (UNED), España

Correo: c.castro.clemente@gmail.com 
Resumen

La violencia entre iguales es un fenómeno complejo de analizar en el que interviene la familia, núcleo de referencia vital para el adolescente, y la escuela, ámbito de pertenencia en el que desarrolla gran parte de su proceso de formación. El objetivo del artículo es profundizar y analizar sus características, clases, fases, síntomas, roles y respuesta que dan estudiantes, profesores y padres ante un suceso de maltrato en el contexto escolar. El acoso tiene efectos devastadores en las víctimas, la ideación suicida es su consecuencia más grave. En cuanto al perfil del agresor, corresponde a una persona líder que desea mantener su estatus de liderazgo en un grupo cada vez mayor. Como conclusión principal en este trabajo se resalta la importancia de compartir, entre la comunidad educativa y los padres, la responsabilidad en la educación y protección de los jóvenes ante situaciones problemáticas de conducta como es la agresión hacia iguales.

\section{Palabras clave}

Acoso - Ciberacoso - Familia - Centro Educativo
Abstract

Peer violence is a complex phenomenon to analyze the family involved, vital core reference for the child, and school, membership in the field of that develops much of their training process. The objective of the study is to deepen and analyze their characteristics, classes, stages, symptoms, roles and response that give students, teachers and parents to an event of violence in the school context. Bullying has devastating effects on victims, suicidal ideation is the most serious consequence. As to the aggressor profile corresponds to a leader who wishes to maintain its leadership status in a growing group. Principal conclusionin this study highlights the importance of sharing, between the education community and parents, responsibility for education and protection of young people in problem situations of conduct as is aggression toward peer.

Keywords

Bullying - Cyberbullying

- Family - School 


\section{Introducción}

La Organización Mundial de la Salud presentó en el año 2002 el Informe mundial sobre la violencia y salud; fue el primer estudio sobre el problema de la violencia a escala mundial. En el documento se definió la violencia como "el uso deliberado de la fuerza física o el poder, ya sea en grado de amenaza o efectivo, contra uno mismo, otra persona o un grupo o comunidad, que cause o tenga muchas probabilidades de causar lesiones, muerte, daños psicológicos, trastornos del desarrollo o privaciones". Asimismo, el informe manifiesta que la violencia está tan presente, que se la percibe a menudo como un hecho ineludible de la condición humana, no hay país ni comunidad a salvo de la violencia. Está en los hogares, escuela, calles, trabajo y otros centros. La incidencia de problemas de conducta y comportamientos irregulares en los adolescentes responden a perfiles y rasgos muy distintos, sin embargo, ninguno está predeterminado a ser violento y tiránico (Gutiérrez y Pernil, 2004).

En general, las investigaciones y estudios realizados sobre la violencia en los jóvenes se han centrado más en el perfil de los agresores y de las víctimas, enfocando la problemática desde una visión individual y personalizada. Pero también cabe abordar el tema desde una visión más social en la que los padres, junto con los maestros y la sociedad en general, tienen parte de responsabilidad (Yuste y Pérez, 2008).

Los niños/niñas y adolescentes están expuestos a la violencia de forma directa o indirecta, no se da tan sólo en la familia o en la calle, también se reproduce en el ámbito escolar. Según Díaz-Aguado (2006), la forma de violencia entre iguales tiene las siguientes características:

- Conductas de diversa naturaleza: burlas, insultos, amenazas, agresiones físicas, asilamiento sistemático, intimidaciones.

- Es un abuso de poder, suele estar provocada por un alumno apoyado, generalmente, en un grupo. 
- Se mantiene en el tiempo por ignorancia o pasividad de las personas cercanas a la víctima sin intervenir directamente.

- Originan problemas que se repiten y prolongan durante cierto tiempo.

Durante la década del 90, irrumpen en la sociedad nuevas Tecnologías de Comunicación e Información (TIC), Internet y teléfonos móviles. Su evolución tecnológica ha sido arrolladora en los últimos años y su omnipresencia en nuestras vidas, característica en la sociedad actual, se ha convertido en algo habitual. Su uso también se ha extendido entre los jóvenes, pero, paralelamente, se han generado nuevas formas de agresión y acoso escolar entre adolescentes, el denominado ciberacoso.

En 1982, en la Declaración sobre Educación de los Medios realizada por la UNESCO, ya se alertaba sobre el poder de los medios de comunicación como un elemento importante de la cultura contemporánea. De igual forma, se resaltaba la importancia en preparar a los jóvenes para vivir en un mundo dominado por imágenes, palabras y sonidos. Preparación cuya responsabilidad debían compartir y asumir, principalmente, la escuela y la familia.

\section{El acoso entre iguales: bullying}

El significado de la palabra bullying procede de la derivación de bull=matón y to bully=intimidar con gritos, amenazas y maltratar a los débiles. Reviste distintas características (Gómez et al, 2007):

- La existencia de una o más conductas de hostigamiento.

- Repetición de la conducta de forma sistemática.

- Se mantiene en el tiempo.

- Afecta a la víctima significativamente en todas las áreas de su vida: académico, afectivo, emocional, familiar. 
- El perfil de la víctima suele corresponder, mayoritariamente, a una persona fuertemente aislada, sin amigos y bastante impopular.

- La victimización puede hacer que aumente más su impopularidad y aislamiento.

Las primeras investigaciones sobre la violencia entre adolescentes en el ámbito escolar se iniciaron en el año 1973 a través del estudio realizado por el Dr. Dan Olweus, quien define el maltrato entre iguales, acoso o bullying, como "comportamiento negativo repetitivo e intencional, desagradable o hiriente de una o más personas dirigido contra una persona que tiene dificultad en defenderse". Es un acto deliberado que implica el uso de la fuerza de forma intencional para hacer daño, conlleva manifestaciones e implicaciones psicológicas y emocionales.

Durante los años 80 y 90 se incrementa el estudio de acoso tras el impacto mediático del suicidio de dos adolescentes en el plazo de una semana en Noruega debido a los reiterados ataques de sus compañeros. Uno de los casos de maltrato relatados por Olweus en su libro (1993:7) refiere a un adolescente de 13 años acosado durante dos años por sus compañeros de clase: "le perseguían por dinero, era obligado a tragarse hierbajos y a beber leche mezclada con detergente, lo golpeaban, le pegaban dentro de los baños y le ponían una cuerda por el cuello para pasearlo como a un animalillo. Cuando los torturadores fueron interrogados sobre estos maltratos dijeron que lo perseguían sólo porque era divertido".

La violencia ejercida sobre la víctima afecta significativamente todos los espacios de su vida. Concurren ciertos comportamientos observables en el adolescente que pueden ayudar a detectar si está siendo acosado. La escuela puede advertir síntomas en los niños, niñas y adolescentes de tristeza, aislamiento, lesiones corporales o heridas, disminución en el rendimiento escolar, poca participación en clase, no ser escogido por otros compañeros para hacer trabajos o para jugar, entre otros. En paralelo, la familia tiene la capacidad de percibir 
indicios de inapetencia, insomnio, depresión, poca comunicación o silencios, depresión, magulladuras, objetos personales rotos y apatía.

Una situación de acoso no es estática, sino que es dinámica y pasa por diferentes etapas. Empieza con pequeños ataques de apariencia inocente a los que la víctima no les da importancia. Gradualmente, las agresiones comienzan a ser más repetitivas e intensas, lo que origina en la víctima malestar emocional, debilidad psicológica y exclusión del grupo (Ruiz, Riuró y Tesouro, 2015). Diferentes investigaciones sobre el acoso entre iguales han pretendido delimitar las fases y características en los procesos y desarrollos de conductas violentas. Sin embargo, ni las fases ni el tiempo determinado para cada una de ellas han podido ser precisadas. Según Molina del Peral y Vecina (2015), aunque las etapas no puedan relacionarse de forma concluyente, sí existe una serie de características que podrían ayudar a determinar la etapa en la que se encuentra el joven victimizado a fin de prevenir la evolución del acoso a etapas superiores:

1. Fase desde los apodos inocentes al estigma social. Representa el inicio del acoso con apariencia de juego entre compañeros. La víctima desea no volver a ser blanco de otro ataque de maltrato sutil.

2. Fase de confusión, acoso y derribo. El acosador ha seleccionado a su víctima y comienza la etapa de hostigamiento a través de continuos insultos, amenazas, chantajes, humillaciones, agresiones, abusos, etc. El acoso, maltrato, que recibe la víctima puede ser físico, verbal, psicológico o social, o darse varios a la vez. La víctima se siente merecedora de ello y vive situaciones de impotencia y soledad que paulatinamente se van incrementando.

3. Aparición de daños psicológicos graves acompañados de sintomatología física. Se producen cambios en la víctima de distinta índole: físicos o somáticos, psicológicos, sociales y de modificación de hábitos.

4. Desenlace final del proceso. El fin a este acoso puede producirse de distintas formas: 
a. La víctima se enfrenta al acosador sin importarle las consecuencias.

b. Autolesiones de la víctima: cortes en las muñecas u otras partes del cuerpo, golpes a sí mismo, etc., y en casos extremos, el suicidio.

\section{El ciberacoso o cyberbullying}

Entre los años 80 y 90 emergen las computadoras, el Internet y los teléfonos móviles, constituyen nuevas Tecnologías de Comunicación e Información. Nuevos contextos de comunicación que suponen un gran impacto en la sociedad por su capacidad de difusión y accesibilidad en todo momento y lugar. Tecnologías que, por sus peculiaridades y características técnicas, repercuten en las actividades y ámbitos económicos, políticos, sociales, educativos, psicológicos y personales. Paralelamente, surge una nueva modalidad de maltrato e intimidación entre niños/niñas y adolescentes, el cyberbullying.

Expresiones violentas que establecen el uso de nuevas formas de intimidación y que se han extendido de manera alarmante entre nuestros jóvenes. Fenómeno que va en aumento y que constituye el uso de nuevas formas de maltrato entre iguales. Los primeros estudios sobre ciberataques comienzan en Estados Unidos en el año 2000 (Finkelhor, Mitchell \& Wolak, 2000; Slonje \& Smith, 2008).

El término ciberacoso, o cyberbullying, es una extensión del maltrato: "acto agresivo e intencionado llevado a cabo de manera repetida y constante a lo largo del tiempo, mediante el uso de formas de contacto electrónicos por parte de un grupo o de un individuo contra una víctima que no puede defenderse fácilmente" (Smith, 2006:3). Esta nueva modalidad de acoso cibernético sustenta unas características específicas:

- Requiere conocimientos sobre el manejo de Internet.

- No es preciso que el agresor tenga más fuerza física que su víctima. 
- Los ataques tienen el propósito de calumniar, desacreditar, denigrar, injuriar, infamar y desprestigiar a la víctima.

- El acoso se hace público y puede desarrollarse en cualquier lugar a través de las TIC.

- Realiza ataques públicos permaneciendo el agresor en anonimato.

- El maltrato se realiza de manera continua y repetitiva.

- No existen espacios donde estar seguros, puede llegar a cualquier lugar, incluso a su casa. Está disponible las 24 horas del día.

El impacto emocional y las consecuencias en las víctimas tienen efectos devastadores: miedo, ansiedad, indefensión, estrés, nerviosismo, trastornos de sueño, dificultad de concentración escolar, frustración, baja autoestima, depresión, irritabilidad, falta de confianza, ira, somatizaciones y como consecuencia más grave, el suicidio (Hinduja \& Patchin, 2010).

La ideación suicida, que comprende desde la idea esporádica de que la vida no merece la pena hasta pensamientos autodestructivos, se incrementa cuando concurren más factores individuales y ambientales como depresión o victimización escolar, entre otros (Rodríguez y Oduber, 2015). Pensamiento negativo y autodestructivo que los jóvenes, víctimas del ciberacoso, contemplan como medida radical y resolutiva ante la situación que viven.

Esta nueva modalidad de maltrato e intimidación entre jóvenes se ha convertido en un problema relevante y de preocupación social por su riesgo impredecible. Fenómeno en aumento que conlleva intencionalidad de hacer daño (aterrorizar, aislar, amenazar, insultar, difamar, etc.), facilita el anonimato del agresor y puede ocurrir lejos de la vista de padres y profesores, lo que limita el control y la ayuda adulta.

En la década del 90 se desarrolla la digitalización de las comunicaciones, el teléfono móvil. Novedosa forma de comunicación que, a través de terminales portátiles de precio asequible, permite la mensajería instantánea. 
La evolución de los teléfonos móviles está en una línea constante de innovación. Ha logrado aumentar su capacidad de transmisión e incluir nuevos servicios como la videoconferencia, posibilidad de grabar imágenes, sonidos, textos, vídeos y descarga de archivos audiovisuales. Su creciente utilización es notoria en casi todos los sectores de la sociedad, incluida en niños de todas las clases sociales y edades (edad cada vez más temprana). Para nuestros adolescentes, su disponibilidad y uso constituyen instrumentos de socialización en el que también hay lugar para la agresión, amenazas y conductas violentas. Entre las vías más utilizadas para ejercer el ciberacoso entre iguales, se destacan: redes sociales (Ask, Tuenti, Twitter, Facebook), mensajería instantánea, acoso a través de fotografías y vídeos de agresiones o humillaciones que se difunden por Internet o teléfono móvil, correo electrónico y videojuegos online.

\section{Las familias}

El núcleo principal de desarrollo psicológico y de apoyo para los niños y adolescentes es la familia, en ella se aprende la mayor parte de las conductas sociales y emocionales. Es una red de comunicación entrelazada donde todos los miembros se influyen entre sí (Fernández y Ponce de León, 2011).

En las últimas décadas, la familia ha experimentado grandes cambios. Emergen nuevos entornos y modelos de convivencia familiares, más libres y plurales. A su vez, la irrupción de repetidas crisis económicas ha impactado en la familia, con padres obligados a trabajar para mantener la economía familiar y, en consecuencia, ha desencadenado en padres sobrecargados y estresados. Situaciones a los que el menor no es ajeno y por las que pueden producirse episodios de carencias afectivas, tensión familiar y desarrollo de comportamientos peligrosos en el adolescente. Nuevos contextos familiares y crisis económicas que repercuten en el tiempo que los padres pueden dedicar a sus hijos y, asimismo, en la manera de afrontar las principales ne- 
cesidades de los adolescentes: su desarrollo, ajuste psicológico y conductas de riesgo (Castro y García, 2013; Castro y Rodríguez, 2016).

La capacidad de los padres en dar respuesta a las necesidades de sus hijos está íntimamente relacionada con el bienestar y desarrollo del menor. Capacidad de atención que puede verse afectada, de forma temporal o permanente y por circunstancias de diversa naturaleza, y provocar déficits de atención a las necesidades de los hijos exponiéndolos a situaciones de riesgo. Del mismo modo, la implicación y tiempo que dediquen los padres a sus hijos tiene una repercusión importante en la probabilidad de desarrollar conductas problemáticas, comportamientos de riesgo y rendimiento del menor. Actualmente, y pese a la existencia de estos riesgos, no se dispone de suficientes estudios que puedan arrojar datos sobre el tiempo, tanto en calidad como en cantidad, que los padres dedican a los hijos (González, Domínguez y Baizán, 2010).

Los padres, en el desarrollo y formación de los hijos ponen en práctica un conjunto de sentimientos, reglas y actitudes con la finalidad de influir, educar y orientar a los hijos para su integración social, proceso denominado como prácticas de crianza o estrategias de socialización. Los estilos de crianza difieren de unos padres a otros y sus efectos en los hijos también son diferentes. Un modelo de crianza con una permisividad excesiva tiene efectos negativos en los hijos, favorece conductas más inmaduras, de escaso autocontrol y falta de independencia y disposición para tomar responsabilidades (Olweus, 1993), mientras que los estilos de crianza democráticos, cercanos y comunicativos favorece la comunicación del adolescente-progenitores. En contra, modelos con una disciplina coercitiva y ausencia de interacciones positivas entre padres-hijos, dificultan el aprendizaje de alternativas en situaciones desajustadas, tiende a mantenerse la continuidad del comportamiento antisocial de los hijos (Hernández, Gómez, Martín y González, 2008). En consecuencia, la familia juega un doble papel, puede ser fuente de bienestar o propiciar manifestaciones violentas y desajustadas entre sus miembros. Todo intento de actuar sobre cualquier tipo de violencia infantil ha de tener en cuenta el contexto familiar y su funcionamiento porque existe una clara rela- 
ción entre los hábitos de crianza y la aparición de conductas agresivas en los hijos (Castro y García, 2013).

Cuando emergieron las nuevas tecnologías digitales en los años 90, el deseo de tener sus propias redes de comunicaciones también se trasladó a nuestros jóvenes. Actualmente se ha convertido en algo cotidiano entre ellos, forman parte de su vida y es una manera de satisfacer muchas de sus necesidades por los recursos que ofrece y dispone: información y relaciones virtuales. Nuevas formas de comunicación en el que también hay lugar para la agresión, la amenaza y la violencia.

Internet es una ventana abierta al mundo y en el que los adolescentes invierten mucho tiempo a la conexión. A pesar de los beneficios que ofrece Internet, no hay que ignorar los posibles riesgos que pueden darse en su uso por los adolescentes: facilidad en el acceso a páginas pornográficas, descarga de archivos que pueden contener software espía o publicitario, blogs gratuitos en el que facilitan mucha información personal, alta con un perfil anónimo o con datos falseados por el adolescente, acoso por extraños o por su grupo de iguales. Peligros que se intensifican si los adolescentes están conectados en soledad y desde la propia habitación. Conjuntamente a estos peligros de uso, muy pocos adolescentes cuentan con alguna supervisión por parte de sus padres, tanto en tiempo de navegación como a los contenidos a los que accede. Los padres se sienten muchas veces limitados en la educación y control de los sitios de acceso porque, a menudo, los jóvenes son más expertos que ellos (Ochaíta, Espinosa y Gutiérrez, 2011).

\section{¿Cómo responden los padres ante una situación de agresión a sus hijos en el centro educativo?}

Distintos autores han analizado la importancia de respuesta que da la familia cuando tienen conocimiento de un episodio de violencia escolar (Fekkes, Pijpers \& Verloove-Vanhorick, 2005; Matsunaga, 2009; Waasdorp \& Bradshaw, 2009; De la Caba y López, 2013). Si 
bien, y en general, los padres no se desentienden ni minusvaloran las situaciones de agresión que pueden experimentar sus hijos, aproximadamente la mitad de las familias pueden desconocer la realidad que viven, situación que depende de los niveles de comunicación y del clima familiar.

Se han realizado diversos estudios a padres de estudiantes con la finalidad de conocer la respuesta que darían si su hijo fuera víctima de una agresión entre iguales en el centro escolar, los resultados revelan una alta predisposición de las familias en colaborar con los centros educativos para intervenir ante los fenómenos de agresión, colaboración condicionada al tipo de acoso que se produzca y a la percepción que la familia tenga sobre la gravedad del hecho.

Según De la Caba y López (2013) ante agresiones directas o relacionales como son revelar secretos, violar la privacidad, aislar, excluir y marginar, el procedimiento que aplican es el de intentar resolver la situación dentro del grupo familiar mediante la elaboración y proposición de alternativas resolutivas. La percepción del conflicto tiende a minimizarlo, quitarle importancia y desentenderse.

Sin embargo, cuando se produce un ataque de ciberacoso, de forma casi generalizada la familia busca apoyo externo, los recursos con los que cuentan les parecen insuficientes para enfrentar esta situación. Las principales instituciones externas a las que acuden, y según respuesta mayoritaria de los padres, son al centro escolar y puestos policiales.

\section{Ámbito educativo}

La escuela, espacio social y de relación en el que, además de los contenidos académicos, proporciona al alumnado una experiencia de relación que adquiere gran trascendencia en los demás espacios sociales. Cambios legislativos y educacionales, nuevas tecnologías, comportamientos de riesgo, desmotivación escolar, crisis de modelos familiares, violencia, etc., influyen en un gran número de menores y 
jóvenes escolarizados en el desarrollo de valores para la vida y para la convivencia.

Dentro del centro educativo, la convivencia escolar es el gran reto, es el escenario social en el que se dan continuas interacciones y en el que el estudiante permanece gran parte de su tiempo. Medios de comunicación, conductas de riesgo, violencia intrafamiliar y violencia social influyen en la escuela, es una multiplicidad de situaciones en el que los niños participan directa o indirectamente. No es un problema reduccionista al colegio, en todas las manifestaciones del menor influye el entorno y, a su vez, interviene en su ámbito educativo. La labor de los tutores es crucial para prevenir comportamientos desajustados y de riesgo, además de la labor principal de impartir formación humana son los que mejor conocen la organización del grupo, relaciones de poder, afectividad, valores, rendimiento escolar y problemas específicos de los alumnos (Castro y Rodríguez, 2016).

El problema de las manifestaciones de violencia en el ámbito educativo, sus consecuencias, tanto para estudiantes como para docentes, es de impotencia, inseguridad, angustia, intranquilidad y desesperanza. Desestabilizan a la comunidad educativa y debilitan las formas instituidas de solidaridad y confianza (Ghiso, 2012).

El profesorado se siente especialmente afectado ante un episodio de violencia escolar en su centro educativo, sienten estar poco preparados para enfrentarlo, les produce insatisfacción en su trabajo, repercute en la sensación de pertinencia y de bienestar de en su ámbito laboral y afecta a su salud mental cuestionándose de forma continua su actuación frente a la conducta de maltrato entre estudiantes (Morales et al., 2014).

Afrontar situaciones de riesgo y de conflicto escolar requiere un trabajo en equipo de todos los colectivos que integran la comunidad educativa para disponer de estrategias, protocolos de actuación, planes de prevención y sistemas de mediación con la finalidad de resolver el conflicto, prevenir conductas de riesgo y generar pautas de buena convivencia. Si estas situaciones no son detectadas en sus fases iniciales, ni a tiempo que permitan poner en marcha una intervención preventiva o reparadora adecuada, pueden agravarse y ocasionar situa- 
ciones de desprotección infantil y juvenil grave, importantes daños emocionales, así como afectar a su desarrollo psíquico-físico.

Los profesionales de Trabajo Social desarrollan un papel importante en la intervención preventiva y asistencial dentro del sistema educativo. En la institución escolar se presentan diferentes situaciones o problemáticas que requieren de un análisis e intervención debidamente planificada, requisitos que reúnen los trabajadores sociales desde su condición de profesionales. Su formación específica, conocimientos adquiridos e interiorizados, metodología de intervención y respuestas adaptadas a las necesidades y realidades, le convierte en el profesional idóneo en la resolución de conflictos escolares.

Funciones que, con frecuencia, son desconocidas por el conjunto de la sociedad y en el que incluso, durante mucho tiempo, sus capacidades y potencialidades se han visto constreñidas hasta el punto de correr el peligro de perder su identidad profesional imponiendo en qué consiste su trabajo (Puyol y Hernández, 2009). Con el transcurso del tiempo, y hasta la actualidad, la participación del trabajador social en la Educación ha ido adquiriendo cada vez una mayor dimensión, no sólo a través de las legislaciones sino también con las numerosas y diferentes metodologías desarrolladas para lograr esa participación en la institución educativa (Ponce de León y Castro, 2014). Su colaboración con el profesorado permite intervenir en las necesidades, dificultades y conflictos en la actividad escolar. No se trata de repartirse los problemas, sino de actuar conjuntamente (Torra, 2009).

La escuela representa, por tanto, uno de los mejores recursos para influir en los comportamientos violentos o disruptivos de los adolescentes. A través de la información, estrategias de prevención y actividades escolares brinda la oportunidad de mejorar los estilos y estrategias de afrontamiento con el objetivo de prevenir posibles alteraciones de tipo emocional, físicas y psicológicos en este grupo de edad (Poch, González, García, Malo y Casas, 2015). 


\section{¿Qué roles pueden desempeñar los estudiantes ante un maltrato entre iguales?}

Cuando se produce un episodio de acoso escolar la principal atención está enfocada hacia las víctimas y agresores, sin embargo, durante el proceso de agresión también están presentes otros alumnos. Las conductas de los estudiantes pueden ser la de intervenir para detener la situación o, por el contrario, permitir, animar o apoyar el hostigamiento.

En el rol que éstos asumen en el maltrato entre iguales pueden diferenciarse los siguientes sujetos (Haro y García, 2014):

- Espectadores: identifican las víctimas y agresores, algunos manifiestan una actitud tolerante mientras que otros muestran indiferencia, toleran la situación. Mayoritariamente se mantienen al margen del maltrato como mecanismos de defensa por temor a ser agredidos ellos también y porque no se sienten con la suficiente confianza en sí mismos de ayudar e intervenir correctamente en la situación.

- Agresor: sujeto carente de empatía hacía el sufrimiento y sentimientos de la víctima, le culpabiliza de la situación. Se siente superior a los demás, no manifiesta sentimientos de culpabilidad ni se avergüenza del maltrato ejercido. En cuanto a sus relaciones interpersonales en el contexto escolar, tiene un comportamiento prosocial, no se observan dificultades de relación, circunstancias que facilitan el sostenimiento de su conducta (Cerezo, Sánchez, Ruiz y Arense, 2015).

- Defensores de la víctima: manifiestan una actitud comprensiva con el menor victimizado, expresan su opinión negativa con sus compañeros ante la situación de maltrato, se sienten avergonzados y culpables ante la conducta de hacer daño deliberado. 


\section{¿Existen diferencias por género y edad en la prevalencia del maltrato?}

En la literatura científica, unos de los factores más estudiados por considerarse fundamentales en la comprensión en el fenómeno de la violencia entre iguales han sido las variables género y edad, las consecuencias del acoso no son iguales para chicos y chicas (Garaigordobil y Aliri, 2013). Sin embargo, no se disponen de suficientes estudios que arrojen diferencias consistentes, incluso los resultados de diferentes investigaciones son contradictorias entre sí.

Algunos trabajos evidencian que el sexo masculino, en comparación con el femenino, participa en mayor proporción como agresores y destacan en conductas de hostigamiento (Calvete, Orue, Estévez, Villardón y Padilla, 2010; Félix-Mateo, Soriano-Ferrer, GodoyMesas y Sancho-Vicente, 2010; Calmaestra, 2011; Buelga y Pons, 2012; Varela, 2012; Save The Children, 2016).

Por el contrario, otros estudios señalan que no hay diferencias significativas en cuanto al género de los agresores (Calmaestra, Ortega y Mora-Merchán, 2008; Garaigordobil y Aliri, 2013; DonosoVázquez, Rubio, Velasco-Martínez y Vilá, 2014; Barón, Buelga y Torralba, 2015).

Respecto a la victimización, las mujeres tienden a ser más agredidas que los varones (Buelga, Cava y Musitu, 2010; Estévez, Villardón, Calvete, Padilla y Orue, 2010; Calmaestra, 2011; Valera, 2012; Garaigordobil y Aliri, 2013; Save The Children, 2013; Vives, Sánchez, Orte y Macías, 2014; Donoso-Vázquez et al., 2014; Save The Children, 2016). En algunos casos, el acoso es por parte de exparejas de una relación afectiva terminada (Donoso-Vázquez et al., 2014).

Por lo que a la edad se refiere, en los cursos de Primaria (alumnos entre los 6 y 12 años de edad), como así revelan varios estudios, ya se empieza a detectar algunas actitudes y prácticas violentas, pero es en Secundaria (estudiantes entre los 12 y 16 años) en los que la violencia se hacen más visible e intensa (Barrigüete 2009; Álvarez-García et al., 2011; Morales y Trianes, 2012; León del Barco et al., 2012; Buelga 
y Pons, 2012; Organización Mundial de la Salud, 2012; Vives et al., 2014; Barón, Buelga y Torralba, 2015; Save The Children, 2016).

Es la etapa de la adolescencia, en el tramo de 12 a 14 años, cuando más prevalece el ciberacoso. Los agresores que utilizan Internet para sus ataques son en su mayoría de $2^{\circ}$ y $3^{\circ}$ de Educación Secundaria Obligatoria (ESO) y las víctimas, de $1^{\circ}$ (12-13 años) y $2^{\circ}$ (13-14 años). Es en $2^{\circ}$ de ESO donde encuentra su momento más álgido, es la etapa más crítica de la violencia a través de las TIC para, posteriormente, decrecer progresivamente (Álvarez-García et al., 2011; Buelga y Pons, 2012; Save The Children, 2016). En $3^{\circ}$ (14-15 años) hay un ligero descenso, pero en $4^{\circ}$ (15-16 años) se manifiesta un claro ascenso en las ciberagresiones.

En general, los alumnos de los primeros niveles de la ESO están más involucrados en malos tratos y en el acoso con teléfono móvil o Internet. Principalmente hacen uso del acoso verbal con el objetivo de causar daño en el círculo de amistades de otra persona o en la percepción de pertenencia al grupo, maltrato relacional (León del Barco et al., 2015; Save The Children, 2016).

¿Cómo actúan los hijos ante una situación de agresión en el centro educativo?

Por lo general, los alumnos primero lo comentan ente ellos, entre amigos, para determinar si es normal o no ese ataque recibido, saber qué piensan ellos y cómo pueden enfrentar la situación. Confían que el centro escolar, principalmente su tutor, y personas adultas con autoridad les protegerán. Sin embargo, domina la "ley del silencio" en los estudiantes cuando se produce un episodio de acoso escolar (Cowie, 2013; Save The Children, 2013).

Cuando el acoso es continuo y repetitivo, no saben a quién recurrir "no tienes ni el qué ni con quién; así que, no sabes qué hacer". Generalmente, el centro escolar no detecta una situación de violencia entre iguales sino es comunicado por otros profesores o por los padres del estudiante victimizado, los alumnos no los comunican "se 
ponen en plan hermético". Sólo en casos de violencia muy extrema se lo comentarían a los profesores, pero no lo hacen de forma voluntaria sino porque los profesionales tienen conocimiento de la situación y se sienten obligados a relatar lo sucedido.

Circunstancias que limitan la intervención de la comunidad educativa y que repercute en el menor produciéndole sentimientos de decepción, angustia y desesperanza. De igual manera, el efecto de esta desilusión y desconfianza potencia su soledad y aislamiento por el problema que está sufriendo y viviendo (Garaigordobil, 2011; Povedano, Cava, Monreal, Varela y Musitu, 2015).

La escuela, al igual que la familia, influye en el desarrollo de valores y comportamientos y representa uno de los mejores recursos para mediar en conductas problemáticas. Desde un trabajo en equipo, conjunto de estrategias y métodos de trabajo favorecen la organización escolar, mejora del comportamiento de los adolescentes y la convivencia del centro. De la misma forma, percibir a los compañeros de clase como amigos y al profesor como adulto de referencia y ayuda, ejerce una importante influencia en su integración social y constructo de identidad (Castro y Rodríguez, 2016).

\section{¿Qué marco normativo dispone España para elaborar estrategias de abordaje ante la conflictividad en las instituciones educativas?}

La sociedad moderna, caracterizada por una cultura de globalización, avance de conocimientos y desarrollo de nuevas tecnologías que emergen con rapidez, están creando nuevas situaciones sociales que no permiten adaptarse suficientemente a la persona y que, colateralmente, demanda un Sistema Educativo flexible que se ajuste a los nuevos contextos. Nuestros jóvenes, nativos digitales, fascinados por todo lo que les ofrece el mundo virtual y las nuevas tecnologías también han encontrado un lugar para el maltrato. Violencia que ha trascendido al entorno escolar y por lo que, en España, tanto en el sistema educativo como en el legislativo, se han dado profundas 
transformaciones con el objetivo de adaptarse a los problemas emergentes y favorecer la protección y cuidado de los adolescentes desde el respeto a los Derechos y Libertades fundamentales.

En España, las competencias educativas se distribuyen entre el Estado, impulsor de las líneas educativas prioritarias, y Comunidades Autónomas, gestoras y legisladoras en su ámbito territorial. Por otra parte, las instituciones educativas disfrutan de autonomía propia en la elaboración, desarrollo y aplicación de los Planes de Convivencia en los que abordan, entre otras materias, protocolos de prevención e intervención ante los casos de bullying y cyberbullying.

En su evolución legislativa en el sistema educativo, tal vez una de la más importantes fue la presentada en el año 1985, Ley Reguladora del Derecho a la Educación. En ella se reconoce el derecho de participación a las asociaciones de padres y Consejos Escolares en la institución educativa y se transfieren competencias de ámbito educativo a las Comunidades Autónomas. No es hasta mediados de la década de los 90 cuando España manifiesta una creciente preocupación en estudiar el deterioro de la convivencia escolar. Desde entonces, los problemas de convivencia y prevención escolar se han convertido en una prioridad que ha dado lugar a la puesta en marcha de distintos programas en términos de investigación como de intervención y políticas nacionales (Pineda-Alfonso y García, 2014).

Así, en estos nuevos contextos, en marzo de 2006 El Ministerio de Educación y Ciencia aprobó el "Plan para la Mejora de la Convivencia Escolar" que, posteriormente, ha sido modificada en el año 2013 por el nuevo "Plan para la Convivencia y Mejora de la Seguridad en los Centros Educativos y sus Entornos".

Los objetivos principales del Plan son los de impulsar hábitos y conductas que promuevan e incrementen la buena convivencia en el entorno escolar y garantizar las normas de funcionamientos y organización en los centros educativos. El programa también aborda el riesgo en menores y jóvenes ante el uso de las TIC e Internet, así como las nuevas tipologías delictivas y formas de intimidación que se han generado. 
Por su parte, las Comunidades Autónomas, y en base al actual Plan, elaboran su Ley de Convivencia y Participación, planes y programas de actuación, así como desarrollo de sus propias normas en la regulación con los derechos y deberes de los alumnos. Conjunto de medidas que las Comunidades Autonómicas publican en sus páginas web para consulta de profesionales, padres, estudiantes o cualquier otro sector interesado. Si bien incluyen medidas preventivas y de intervención, no son comunes entre las Comunidades ni son de obligatoria implantación como tampoco abordan de igual manera los fenómenos del acoso y ciberacoso.

Respecto a las instituciones escolares, con un marcado interés en favorecer la convivencia escolar, se involucran en conocer y analizar los factores de riesgo que pueda incidir en la construcción de un buen clima educativo. En consecuencia, en las últimas décadas se han impulsado distintos procedimientos de coordinación y cooperación con las instituciones públicas a fin de intercambiar conocimiento y facilitar recursos que permitan, no sólo alcanzar el nivel de conocimiento adecuado del estudiante, sino también abordar las nuevas situaciones problemáticas emergentes como es el acoso escolar. Sin embargo, la autonomía que disfrutan los centros educativos en la elaboración, desarrollo y aplicación de su propios Planes de Convivencia ha generado una gran diversidad de protocolos de prevención e intervención ante los casos de maltrato entre iguales no comunes, pero sí similares, entre el resto de instituciones educativas.

\section{Conclusiones}

La violencia no se da tan sólo en la calle o en la familia, también se reproduce en el ámbito escolar, entre iguales. Burlas, insultos, amenazas, agresiones físicas, asilamiento sistemático e intimidaciones originan graves daños en el adolescente y pueden prolongarse durante cierto tiempo por ignorancia o pasividad de las personas cercanas a la víctima sin intervenir directamente. En las manifestaciones de maltrato entre iguales se dan una serie de factores interrelacionados 
entre sí como son la autoestima, familia, escuela, relación entre iguales y amistad que influyen en los menores y en la expresión de sus conductas, equilibradas o inadaptadas (Castro y Rodríguez, 2016). Es un cúmulo de situaciones que, si no son detectadas en su fase inicial, pueden poner en riesgo al menor y contribuir al desarrollo de disfunciones físicas y psíquicas.

Con la irrupción de la TIC emergió una nueva forma de maltrato en la juventud, el ciberacoso. En la actualidad, la disponibilidad y uso de las nuevas Técnicas de Información y Comunicación constituyen instrumentos de socialización para nuestros adolescentes, pero en él, también hay lugar para la agresión, amenazas y conductas violentas. Entre los ataques más frecuentes ejercidos por los agresores en Internet destacan publicar fotos de la víctima en las redes sociales con etiquetados o comentarios hirientes, descalificaciones e insultos y ataques emocionales ofensivos con intención de ridiculizar y aislar a la víctima.

Emergente forma de maltrato que revela la importancia de la actuación preventiva y que exigen alternativas de intervención actualizadas y rigurosas. Trabajo que corresponde a la comunidad educativa, familia e instituciones a través de una coordinación de esfuerzos.

El papel de las familias representa un recurso clave para prevenir y afrontar la violencia. El contexto familiar puede reducir estos factores de riesgo, aunque, en paralelo, existen otras situaciones de distinta naturaleza e intensidad que pueden mermar la atención, el cuidado y la educación que la familia procura a sus hijos y que pueden desencadenar en déficits de atención a sus necesidades: horarios laborales extensos, conciliación laboral, padres estresados, implicación y tiempo de dedicación a los hijos.

La familia es una red de comunicación entrelazada donde todos los miembros se influyen entre sí (Fernández y Ponce de León, 2011). Dispone de una estructura que atiende a la interacción interna de sus integrantes y a la interacción externa con el medio que les rodea (Bronfenbrenner, 1979). Dentro de esta organización, en el menor influye el clima familiar en el que se encuentre y las situaciones que observa en relación a malos tratos o conflictos, grado de agresividad, 
intensidad, frecuencia, duración y resolución final. Circunstancias captadas y percibidas por los hijos que, a largo plazo, pueden impactar en su desarrollo y en la forma de afrontar las situaciones o problemas aceptando e interiorizando la violencia como la única manera de resolverlos (Castro y García, 2013). Como refiere Bandura (1986) sobre la teoría del aprendizaje vicario-social, la adquisición de conductas agresivas se puede aprender a partir de la observación y la imitación de modelos, los menores pueden llegar a aceptar e interiorizar la violencia considerándola como "normal" y como medio apropiado para obtener lo que desea o como solución ante los problemas que se les pueda plantear con sus semejantes.

Frente a estas situaciones desajustadas, numerosas investigaciones han comprobado que, según el estilo de crianza desarrollado en el contexto familiar, favorecerá o disminuirá la manifestación de comportamientos problemáticos en los adolescentes. Consecuentemente, se subraya la importante trascendencia en mantener una relación afectiva entre padres-hijos que interpreten y respondan a las señales emocionales de los hijos para lograr una sintonía emocional que impida el desarrollo de conductas problemáticas en los niños.

La escuela no es ajena a estas manifestaciones de actitudes desajustadas en los adolescentes. Dentro del centro educativo, la convivencia escolar es el gran reto, es el escenario social en el que se dan continuas interacciones y en el que estudiante permanece gran parte de su tiempo. Constituye el espacio social en el que los niños viven multiplicidad de situaciones, en él aprenden a convivir e interiorizar los valores sociales y humanos (Castro y Rodríguez, 2016).

El conflicto, es algo natural en el ámbito escolar pero cuando surge un episodio de maltrato ente iguales, esta situación afecta negativamente a estudiantes, profesores y progenitores, desestabilizan a la comunidad educativa, a la familia y debilitan las formas instituidas de solidaridad y confianza (Ghiso, 2012). Afrontar situaciones de acoso entre iguales requiere una intervención especial ligada a un conjunto específico de capacitaciones, conocimientos, destrezas y herramientas que posibiliten mediar en los diversos contextos y entornos más cercanos al menor en el que el conflicto se presenta (Morales et al., 
2014). Competencias y capacidades que ofrecen los profesionales de Trabajo Social al sistema educativo. Las grandes transformaciones sociales y escolares que se han experimentado en las últimas décadas, evidencian la importante labor del trabajador social frente al reto de detectar las necesidades de cada época, adaptar su intervención a los problemas coyunturales y conocer de primera mano la situación de las familias y de la persona a fin de mediar y resolver múltiples situaciones que permitan una eficaz intervención en la educación.

Si bien el centro educativo, junto con la familia, representan las dos instituciones básicas que más influyen en la prevención de conductas desajustadas y disruptivas de los adolescentes, también es necesaria la respuesta y participación de los organismos públicos en materia de seguridad de la población juvenil. Los niños y niñas tienen mayores posibilidades de modificar determinados comportamientos, pero esto sólo es posible si existe un sistema público apropiado, diferente al de los adultos, que ofrezca respuestas a las nuevas demandas y situaciones problemáticas dentro del sistema educativo a fin de que puedan favorecer la convivencia escolar.

En España, las competencias educativas se distribuyen entre el Estado y Comunidades Autónomas. Asimismo, las instituciones escolares disfrutan de autonomía propia en la elaboración, desarrollo y aplicación de los Planes de Convivencia y protocolos de prevención e intervención ante los casos de maltrato entre iguales, entre otras funciones. Competencias compartidas que generan una pluralidad normativa y protocolaria ante los casos de maltrato escolar entre iguales. Dispersión procedimental que obstaculizan la elaboración e implementación de programas de prevención a nivel nacional.

No se trata sólo de realizar cambios legislativos y elaborar planes y programas en materia educativa, ni eliminar los conflictos puntuales, sino dotar a toda la comunidad escolar de un conjunto de protocolos de acción que les permita afrontar y manejar situaciones conflictivas. Es necesario, desde un trabajo en equipo, proporcionar un conjunto de estrategias y métodos de trabajo comunes que favorezcan la organización educativa, incidan en la mejora del comportamiento de los adolescentes y convivencia escolar fomentando, a su vez, la 
participación de estudiantes, familias y profesorado en cuestiones relacionadas con la seguridad y prevención de la violencia entre iguales.

\section{Referencias bibliográficas}

ÁlVAREZ-GARCÍA, D., NÚÑEZ, J.C., ÁLVAREZ, L., DOBARRO, A., RODRÍGUEZ, C., Y GONZÁLEZ-CASTRO, P. "Violencia a través de las tecnologías de la información y la comunicación en estudiantes de secundaria", en: Revista Anales de Psicología, 27, 2011. Pp. 221-231.

BANDURA, A. Social foundations of thought and action: A social cognitive theory. Nueva Jersey, Prentice-Hall, 1986.

BARÓN, J.O., BUELGA, S., Y TORRALBA, E. “Simptomatologia depressiva, estrès percebut i suport social en adolescents víctimes de ciberassetjament”, en: Revista Anuari de psicologia de la Societat Valenciana de Psicologia, 16, 2015. Pp.91-104.

BARRIGÜETE, L. "El profesorado ante la violencia y los conflictos escolares", en: Revista Educatio siglo XXI, 27, 2009. Pp. 207-232.

BRONFENBRENNER, U. La ecología del desarrollo humano. Barcelona, Paidós, 1979.

BUELGA, S. Y PONS, J. “Agresiones entre adolescentes a través del teléfono móvil y de Internet”, en: Revista Psychosocial Intervention, 21, 2012. Pp. 91-101.

BUELGA, S., CAVA, M.J., Y MUSITU, G. "Cyberbullying: victimización entre adolescentes a través del teléfono móvil y de Internet”, en: Revista Psicothema 4, 2010. Pp. 784-789.

CALMAESTRA, J. Cyberbullying: prevalencia y características de un nuevo tipo de bullying indirecto. Tesis doctoral. Facultad de Ciencias de la Educación. Departamento de Psicología. Universidad de Córdoba, España, 2011.

CALMAESTRA, J., ORTEGA, R., Y MORA-MERCHÁN, J.A. "Las TIC y la convivencia: un estudio sobre formas de acoso en el ciberespacio", en: Revista Investigación en la Escuela, 64, 2008. Pp.93-104.

CALVETE, E., ORUE, I., ESTÉVEZ, A., VILLARDÓN, L., Y PADILlA, P. "Cyberbullying in adolescents: Modalities and aggressors" profile", en: Revista Computers in Human Behavior, 26, 2010. Pp.1128-1135.

CASTRO, C. Y GARCÍA, F.J. "La influencia de la familia en el desarrollo y adquisición de actitudes violentas y agresivas en la infancia y juventud", en: Revista Trabajo Social Hoy, 69, 2013. Pp. 21-30. 
CASTRO, C. Y RODRÍGUEZ, E. "Intervención social con adolescentes: Necesidades y recursos”, en: Revista Trabajo Social Hoy, 77, 2016. Pp. 7-23. DOI 10.12960/ TSH.2016.0001.

CEREZO, F., SÁNCHEZ, C., RUIZ, C., Y ARENSE, J.J. "Roles en bullying de adolescentes y preadolescentes, y su relación con el clima social”, en: Revista de Piscodidáctica, 20 (1), 2015. Pp. 139-155.

COWIE, H. "El impacto emocional y las consecuencias del ciberacoso", en: Revista CONVIVES, 3, 2013. Pp. 16-24.

DE LA CABA, M.A. Y LÓPEZ, R. "Las respuestas de los padres ante situaciones hipotéticas de agresión a sus hijos en el contexto escolar", en: Revista de Educación, Extraordinario, 2013. Pp. 236-260.

DÍAZ-AGUADO, M. El acoso escolar y la prevención de la violencia desde la familia. Madrid, Consejería de Familia y Asuntos Sociales Comunidad Autónoma de Madrid, España, 2006.

DONOSO-VÁZQUEZ, T., RUBIO, M.J., VELASCO-MARTÍNEZ, A., Y VILÁ, R. "Ciberacoso en función del género: propuestas de análisis", en: V Congreso Universitario Internacional Investigación y Género. Sevilla, España, 2014. Pp. 873-892.

ESTÉVEZ, A., VILLARDÓN, L., CALVETE, E., PADILLA, P., Y ORUE, I. "Adolescentes víctimas de cyberbullying: prevalencia y características", en: Revista Behavioral Psychology / Psicología Conductual, 18, 2010. Pp. 73-89.

FEKKES, M., PIJPERS, F.I.M., \& VERLOOVE-VANHORICK, S.P. “Bullying: Who Does What, When and Where? Involvement of Children, Teachers and Parents in Bullying Behavior, en: Revista Health Education Research, 20, 2005. Pp. 81-91.

FÉLIX-MATEO, V., SORIANO-FERRER, M., GODOY-MESAS, C., Y SANCHOVICENTE, S. "El ciberacoso en la enseñanza obligatoria", en: Revista Aula Abierta, 38, 2010. Pp. 47-58.

FERNÁNDEZ, T. Y PONCE DE LEÓN, L. Trabajo Social con familias. Madrid, Ediciones Académicas, S.A, 2011.

FINKELHOR, D., MITCHELL, K.J., \& WOLAK, J. Online victimization: A report on the nation's youbt. Alexandria: VA: Washington, D.C. National Genter for Missing and Exploteid Children, 2000.

GARAIGORDOBIL, M. "Bullying y Cyberbullying: Conceptualización, prevalencia y evaluación”, en: Revista FOCAD, 2, 2011. Pp. 1-22.

GARAIGORDOBIL, M. Y ALIRI, J. "Ciberacoso (“cyberbullying”) en el país vasco: diferencias de sexo en víctimas, agresores y observadores”, en: Revista Behavioral Psychology / Psicología Conductual, 3, 2013. Pp. 461-474. 
GHISO, A. "Algunos límites de las respuestas frente a la violencia y la inseguridad, en las instituciones educativas", en: Revista Latinoamericana de Ciencias Sociales, Niñez y Juventud, 10, 2012. Pp. 815-824.

GÓMEZ, A., GALA, F.J., LUPIANI, M., BERNALTE, A., MIRET, M.T., LUPIANI, S. Y BARRETO, M.C. "El "bullying" y otras formas de violencia adolescente" en: Revista Cuad Med Forense 13, 2007. Pp.165-177.

GONZÁLEZ, M.J., DOMÍNGUEZ, M. Y BAIZÁN, P. “Cuidado parental en la infancia y desigualdad social: un estudio sobre la Encuesta de Empleo del Tiempo en España”, en: Revista Laboratorio de Alternativas, 158, 2010. Pp. 5-56.

GUTIÉRREZ, A. Y PERNIL, P. Historia de la infancia: Itinerarios educativos. Madrid, UNED, 2004.

HARO, I. Y GARCÍA, B. "Variables emocionales y sociomorales asociadas con el tipo de rol que asumen los alumnos y alumnas en el maltrato entre iguales", en: Revista Apuntes de Psicología, 1, 2014. Pp. 15-23.

HERNÁNDEZ, M., GÓMEZ, I., MARTÍN, M.J., Y GONZÁLEZ, C. "Prevención de la violencia infantil-juvenil: estilos educativos de las familias como factores de protección", en: Revista International Journal of Psychology and Psychological, 8, 2008. Pp. 73-84.

HINDUJA, S. \& Patchin, J.W. "Bullying, Ciberbullying and Suicide", en: Revista Archives of Suicide Research, 14, 2010. Pp. 206-221.

Jefatura del Estado de España. (23 de abril de 2016). Ley Orgánica 8/1985, de 3 de julio reguladora del Derecho a la Educación. Obtenido de Boletín Oficial del Estado de España, 159: https://www.boe.es/boe/dias/1985/07/04/pdfs/A21015-21022.pdf

LEÓN DEL BARCO, B., FELIPE-CASTAÑO, E., POLO DEL RÍO, M.I., Y FAJARDOBULLÓN, B. "Aceptación-rechazo parental y perfiles de victimización y agresión en situaciones de bullying", en: Revista Anales de Psicología, 31, 2015. Pp. 600-606.

MATSUNAGA, M. "Parents Don't (Always) Know Their Children Have Been Bullied: Child-Parent Discrepancy on Bullying and Family-Level Profile of Communication Standards”, en: Revista Human Communication Research, 35, 2009. Pp. 221-247.

Ministerio de Educación y Ciencia de España. (16 de julio de 2016). Plan para la mejora de la convivencia escolar 2006. Obtenido de http://www.mecd.gob.es/ $\mathrm{dctm} / \mathrm{ministerio/educacion/sistema-educativo/politicas/2010-acuerdo-marco-2.}$ pdf?documentId=0901e72b800bb84c

Ministerio del Interior de España. (14 de julio de 2016). Instrucción 7/2013 de la Secretaría de Estado de Seguridad, sobre el "Plan Director para la Convivencia y Mejora de la Seguridad en los Centros Educativos y sus Entornos”. Obtenido de http://www.interior.gob.es/documents/642012/1568685/Instruccion_7_2013.pdf/ cef1a61c-8fe4-458d-ae0d-ca1f3d336ace

MOLINA DEL PERAL, J.A. Y VECINA, P. Bullying, ciberbullying y sexting. Madrid, Ediciones Pirámide (Grupo Anaya S.A.), 2015. 
MORALES, F.M. Y TRIANES, M.V. "Análisis de valores y actitudes en temas morales en estudiantes de Educación Secundaria”, en: Revista Psicología Educativa, 18, 2012. Pp. 65-77.

MORALES, M., LÓPEZ, V., BILBAO, M.A., VILLALOBOS, B., OYARZÚN, D., OLAVARRÍA, D. y ASCORRA. P. "El papel mediador de la capacitación docente en el manejo de la violencia escolar sobre el bienestar social de profesores", en: Revista Terapia Psicológica, 3, 2014. Pp. 217-226.

OCHAÍTA, E., ESPINOSA, M.A., Y GUTIÉRREZ, H. "Las necesidades adolescentes y las nuevas tecnologías de la información y la comunicación”, en: Revista de Estudios de Juventud 92, 2011. Pp. 87-110.

OLWEUS, D. Hackkycklingar och oversittare: forskning orn skol-mobbing. Estocolmo, Almqvist \& Wiksell, 1973.

OLWEUS, D. Bullying oat school: What we know and what we can do. Oxford, Blackwell Publishers, 1993.

ORGANIZACIÓN MUNDIAL DE LA SALUD. Informe mundial sobre la violencia y salud: resumen. Washington D.C., Organización Panamericana de la Salud, 2002.

ORGANIZACIÓN MUNDIAL DE LA SALUD. Protección y seguridad en internet: retos y avances en los Estados miembros: basado en los resultados de la segunda encuesta mundial sobre cibersalud. Organización Mundial de la Salud, 2012.

PINEDA-ALFONSO, J.A. Y GARCÍA, F.F. "Convivencia y disciplina en el espacio escolar: discursos y realidades”, en: Revista Electrónica de Geografía y Ciencias Sociales, 496, 2014. Pp. 1-21.

POCH, F., GONZÁLEZ, M., GARCÍA, Y., MALO, S., Y CASAS, F. “Los estilos y estrategias de afrontamiento y su relación con el bienestar personal en una muestra de adolescentes”, en: Revista Anales de Psicología, 1, 2015. Pp. 226-233.

PONCE DE LEÓN, L. Y CASTRO, C. "El desarrollo de la Investigación en el Trabajo Social en España. Comparativa con la Educación Social”, en: Revista Ehquidad. International Welfare Policies and Social Work Journal, 2, 2014. Pp. 143-157. DOI 10.15257/ehquidad.2014.0012.

POVEDANO, A., CAVA, M.J., MONREAL, M.C. VARELA, R., Y MUSITU, G. "Victimización, soledad, violencia manifiesta y relacional en la escuela desde una perspectiva de género", en: Revista International Journal of Clinical and Health Psychology, 1, 2015. Pp. 44-51.

PUYOL, B. Y HERNÁNDEZ, M. “Trabajo Social en educación”, en: Revista Qurriculum, 22, 2009. Pp. 97-117.

RODRÍGUEZ, J.A. Y ODUBER, J.A. "Ideación suicida y grupo de iguales: análisis en una muestra de adolescentes venezolanos", en: Revista Universitas Psychologica, 14, 2015. Pp. 1129-1140. 
RUIZ, R., RIURÓ, M., Y TESOURO, M. "Estudio del bullying en el ciclo superior de Primaria”, en: Revista Educación XXI, 18.1, 2015. Pp. 345-368.

SAVE THE CHILDREN ESPAÑA. Acoso escolar y ciberacoso: propuestas para la acción. España, Save The Children, 2013.

SAVE THE CHILDREN ESPAÑA. Yo a eso no juego. Estudio Bullying y Cyberbullying en la infancia. España, Save The Children, 2016.

SLONJE, R. \& SMITH, P.K. “Cyberbullying: Another main type of bullying?”, en: Revista Scandinavian Journal of Psychology, 49, 2008. Pp.147-154.

SMITH, P. "Ciberacoso: naturaleza y extensión de un nuevo tipo de acoso dentro y fuera de la escuela”, en: Congreso Educación. Palma de Mallorca, España, 2006. Pp.1-17.

TORRA, M. "El Trabajo Social en la enseñanza”, en: Revista de Servicios Sociales y Política Social, 86, 2009. Pp.125-128.

UNESCO. International Symposium on Education of the Public in the Use of Mass Media: Problems, Trends and Prospects. Grünwald, Alemania, UNESCO, 1982.

VARELA, R. Violencia, Victimización y Cyberbullying en adolescentes escolarizados/ as: una perspectiva desde el Trabajo Social. Tesis doctoral, Facultad de Ciencias Sociales. Departamento de Trabajo Social y Servicios Sociales, Universidad Pablo Olavide, Sevilla, 2012.

VIVES, M., SÁNCHEZ, L., ORTE, C., Y MACÍAS, L. "El Cyberbullying. Conocer para actuar”, en: Revista INNODOCT, 2014. Pp.1-10.

WAASDORP, T.E. \& BRADSHAW, C.P. "Child and Parent Perceptions of Relational Aggression within Urban Predominantly African American Children's Friendships: Examining Patterns of Concordance", en: Revista Journal of Child and Family Studies, 18, 2009. Pp. 731-745.

YUSTE, N. Y PÉREZ, M.C. "Las cuestiones familiares como causa de la violencia escolar según los padres", en: Revista European Journal of Education and Psychology, 1, 2008. Pp. 19-27.

Recepción: 23/03/2016

Aceptación: 14/09/2016 